\title{
MVDR algorithem for wideband coherent source signals based on data reconstruction
}

\author{
Hui Xia ${ }^{1}$ \\ Software College,Shenyang Normal university, Shenyang, 110034, China \\ freund_xia@126.com
}

\begin{abstract}
Keywords: coherent source signals; MVDR algorithem; data reconstruction
Abstract. Minimum Variance Distortionless Response(MVDR) algorithm for wideband coherent signals based on data reconstruction is proposed.Based on the sampling theorem, the output of different array elements at the same time is taken as the sampling of the continuous line array, and the data of the time domain is re-sampled. The method is used to estimate the azimuth of the broadband coherent source and a method of obtaining the focus error is proposed to analyze the performance of the MVDR algorithm of the wideband coherent source. Computer simulation of the FFT interpolation method and data reconstruction method of the azimuth estimation results show that for the broadband MVDR algorithm, data reconstruction method than the FFT interpolation method has better resolution probability and lower RMS error.
\end{abstract}

\section{Introduction}

Broadband signal processing in the sonar, radar, array microphones and other fields has a wide range of applications. The common methods of broadband signal processing include non-coherent signal subspace method [1] (ISM) and coherent signal subspace method [2] (CSM). The ISM method is computationally large and can not handle coherent signals. By focusing, the observations at different frequencies are aligned on the subspace of a certain frequency to obtain the observations of the focussed synthesis and the azimuth estimation is carried out on this basis. Because the CSM method needs to estimate the DOA and its performance is influenced by the estimated azimuth, the spatial interpolation method $[3,4,5]$ is proposed to avoid the azimuth prediction problem. Subspace-based high-resolution algorithms [6,7] for azimuth estimation usually need to estimate the number of sources.

In this paper, the time-domain data reconstruction method is introduced into the space-domain data, and the narrow-band MVDR algorithm is used to estimate the azimuth of the broadband coherent sources. The practical steps of the MVDR algorithm are given. The simulation results show that the data reconstruction method has more superior azimuth estimation performance than the FFT interpolation method for wideband MVDR algorithm.

\section{Broadband Signal Model}

An array of $M$ elements is arranged to receive $P$ wideband stationary random signals located at the far field. When there is additive noise, the signal received by the $m$ th element can be expressed as:

$\boldsymbol{x}_{m}(t)=\sum_{p=1}^{p} \boldsymbol{s}_{p}\left[t-\tau_{m}\left(\theta_{p}\right)\right]+\boldsymbol{n}_{m}(t)$

Where $m=1,2 \ldots, M, p=1,2 \ldots, P, s_{p}(t)$ is the $p$ th source, $\boldsymbol{n}_{m}(t)$ is additive noise on the $m$ th element, $\theta_{p}$ is the azimuth of the $p$ th signal, and in the uniform line array, $\tau_{m}\left(\theta_{p}\right)=(m-1) d \sin \left(\theta_{p}\right) / c$, denotes the propagation delay of the $p$ th signal (relative to the reference point) received by the $m$ th element, and $d$ is the spacing of the elements.

In the frequency domain, eq.(1) can be expressed as:

$$
\boldsymbol{X}\left(\omega_{j}\right)=\boldsymbol{A}\left(\omega_{j}, \gamma\right) \boldsymbol{S}\left(\omega_{j}\right)+\boldsymbol{N}\left(\omega_{j}\right)
$$

where 


$$
\begin{aligned}
& \left.\boldsymbol{X}\left(\omega_{j}\right)=\left[\begin{array}{lllll}
\boldsymbol{X}_{1}\left(\omega_{j}\right) \boldsymbol{X}_{2} & \left(\omega_{j}, \ldots, \boldsymbol{X}_{M}\right. & \phi_{j}
\end{array}\right]\right), \\
& \boldsymbol{A}\left(\omega_{j}, \gamma\right)=\left[\boldsymbol{a}\left(\omega_{j}, \theta_{1}\right) \boldsymbol{a}\left(\phi_{j}, \theta_{2}, \ldots, \boldsymbol{a} \phi_{j}, \theta_{p}\right]\right) \\
& \left.\boldsymbol{S}\left(\omega_{j}\right)=\left[\begin{array}{lllll}
\boldsymbol{S}_{1}\left(\omega_{j}\right) \boldsymbol{S}_{2} & \left(\omega_{j}, \ldots, \boldsymbol{S}_{P} \phi_{j}\right.
\end{array}\right]\right), \\
& \left.N\left(\omega_{j}\right)=\left[\begin{array}{lll}
N_{1}\left(\omega_{j}\right) N_{2} & \left(\omega_{j}, \ldots, \ldots, N_{M}\right. & \phi_{j}
\end{array}\right]\right),
\end{aligned}
$$

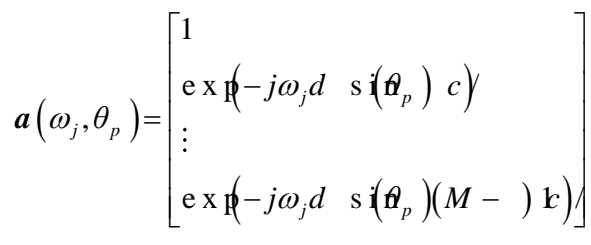

where $\boldsymbol{X}_{m}\left(\omega_{j}\right) 、 S_{m}\left(\omega_{j}\right)$ and $\boldsymbol{N}_{m}\left(\omega_{j}\right)$ is frequency domain form of observation vector $\boldsymbol{x}_{m}(t)$ 、 signal vector $\boldsymbol{s}_{p}(t)$ and noise vector $\boldsymbol{n}_{m}(t)$ at the frequency $\omega_{j}$ respectively, $\boldsymbol{a}\left(\omega_{j}, \theta_{p}\right)$ is direction vector, $\mathrm{T}$ denotes indicates transpose, $m=1,2 \ldots, M, p=1,2 \ldots, P, j=1,2, \ldots J, J$ is the number of frequency points to be processed.

\section{Data reconstruction method}

Since the signal subspace of a wideband signal is a function of frequency and signal source orientation, the signal subspaces of different frequencies are different from each other. In order to align the signal sub-spaces of each sub-band, it is necessary to linearly transform the array output so that the direction matrix at different frequencies within the bandwidth range is kept constant. Then the element spacing $d$ should be a function of frequency, set the focus frequency $f_{0}$, should be:

$$
d\left(f_{j}\right) f_{j}=d f_{0}
$$

In order to obtain the output of the virtual array which is different from the actual array element spacing, the real array must be interpolated.

For uniform line arrays, interpolation can be performed using the sampling theorem. As can be seen from the sampling theorem, if the continuous-time signal $s(t)$ is of limited bandwidth $\left(|\omega|<\omega_{m}\right)$, the continuous signal can be completely determined by the sampling value $s\left(n T_{s}\right)$ at its discrete interval (sampling interval $T_{s}$ satisfies the sampling theorem). We can reconstruct $s(t)$ by generating a periodic impulse string whose impulse amplitude is the successive samples and then passing the impulse train through a low-pass filter with a cut-off frequency $\omega_{c}$ (which satisfies $\left.\omega_{m}<\omega_{\mathrm{c}}<\omega_{s}-\omega_{m}\right)$ The output of the filter is $s(t)$.

Its reconstruction formula is:

$$
s(t)=\sum_{n=-\infty}^{n=+\infty} s\left(n T_{s}\right) \cdot \frac{\sin \left[\omega_{c}\left(t-n T_{s}\right) / \pi\right]}{\omega_{c}\left(t-n T_{s}\right) / \pi}
$$

The formula is applied to the array element output of the uniform line array, that is, the output of each array element at the same time is regarded as sampling to the continuous linear array. At this time, the sampling value $s\left(n T_{s}\right)$ and sampling interval $T_{s}$ to meet $T_{s}=d$, corresponds to the output of each array element.

As long as the spatial sampling theorem is satisfied, that is, $d / \lambda_{\min } \leq 0.5$ ( $\lambda_{\min }$ is the wavelength corresponding to the highest frequency of the signal), the output of any point on the continuous linear array can be recovered from the output of each element at that moment.

In the actual situation, can not satisfy $n \in(-\infty,+\infty)$, adopt the following method to realize spatial data reconfiguration:

1) The subband data matrix $X_{B}$ at each frequency point is obtained by subband decomposition of the received array data.

2) The minimum frequency $f_{l}$ of the selected signal is taken as the focusing frequency. To make each subband data converge to the same frequency $f_{l}, x_{B}$ is reconstructed after the data matrix $X_{B I}$ sampling interval:

$$
T_{s}^{\prime}=T_{s} \cdot f_{l} / f(j)=d \cdot f_{l} / f(j)
$$

where $f(j)$ is the signal frequency corresponding to the $j$ th frequency point, $j=1,2, \cdots J, J$ is the number of frequency points to be processed, so the data length of $X_{B I}$ is:

$$
M^{\prime}=(M-1) d / T_{s}^{\prime}=(M-1) f(j) / f_{l}
$$


3) After reconstructing the spatial data,we get:

$$
\boldsymbol{X}_{\boldsymbol{B} I}=\boldsymbol{X}_{\boldsymbol{B}} \cdot \frac{\sin \left(\omega_{c}\left(\boldsymbol{E} 1 \times \boldsymbol{T}-\boldsymbol{T}_{0} \times \boldsymbol{E} 2\right) / \pi\right)}{\omega_{c}\left(\boldsymbol{E} 1 \times \boldsymbol{T}-\boldsymbol{T}_{0} \times \boldsymbol{E} 2\right) / \pi}
$$

where $\boldsymbol{E}_{M \times 1}=[1,1, \cdots 1]^{\mathrm{T}}, \quad \boldsymbol{E} \boldsymbol{2}_{1 \times N}=[1,1, \cdots 1], \quad N$ is length of vector $\boldsymbol{T}, \quad \boldsymbol{T}_{0}=[0, d, \cdots,(M-1) d]^{\mathrm{T}}, \quad \boldsymbol{T}=\left[0, T_{s}^{\prime}, \cdots, M^{\prime} T_{s}^{\prime}\right]$, $(\cdot)^{\mathrm{T}}$ denotes transpose; $\omega_{c}$ satisfy $\omega_{m}<\omega_{\mathrm{c}}<\omega_{s}-\omega_{m}$, and $\omega_{s}=2 \pi / d$.

4) The matrix of $M$ rows in $X_{B I}$ is selected as the interpolated matrix, and then the covariance matrix $R$ is calculated.

5) The covariance matrix corresponding to $J$ frequency points is obtained according to the above steps, then the mean value $\overline{\boldsymbol{R} x}$ is obtained. Then the MVDR algorithm is used to estimate the DOA and the spatial azimuth spectrum of the target is obtained.

$$
P_{M V D R}=\frac{1}{\boldsymbol{a}\left(f_{0}, \theta\right)^{\mathrm{H}} \operatorname{inv}(\overline{\boldsymbol{R} x}) \boldsymbol{a}\left(f_{0}, \theta\right)}
$$

where $\operatorname{inv}(\cdot)$ and $(\cdot)^{\mathrm{H}} \mathrm{d}$ enote inversion operations and conjugate transpose, respectively, $a\left(f_{0}, \theta\right)$ represents the direction vector at the focus frequency.

\section{Focusing error}

Focusing error is the main factor to determine the performance of the MVDR algorithm. For the conventional wide-band focusing method, the principle of focusing error is given in. However, since the spatial resampling method does not need to solve the focusing matrix, The focus error is obtained by the previous method. Therefore, a new principle of focusing error is proposed.

$$
\varepsilon_{j}=\frac{1}{M}\left\|\boldsymbol{F}_{X 0}-\boldsymbol{F}_{X j}\right\|_{F}
$$

Where, $\mathrm{M}$ is the number of elements, $\boldsymbol{F}_{X 0}$ and $\boldsymbol{F}_{X j}$ are the matrix of $M \times 1, \boldsymbol{F}_{X 0}$ represents the frequency domain data under the focus frequency, $\boldsymbol{F}_{X_{j}}$ represents the frequency domain data after the decoherence processing at frequency $f_{j}, j=1,2, \cdots, J, \mathbf{J}$ is the number of frequency points to be processed, and \|\|$_{F}$ the Frobenious norm.

\section{Test results}

Simulation environment: Consider a uniform line array composed of 16 array elements, with the element spacing being half of the wavelength corresponding to the highest frequency of the signal. The noise is the uncorrelated spatial white noise, the center frequency of the two coherent incident signals is $f_{0}=800 \mathrm{~Hz}$, the relative bandwidth is $50 \%$, the second signal is the first signal delay $0.05 \mathrm{~s}$, the angle of incidence is $-2^{\circ}, 4^{\circ}$, sampling frequency $f_{s}=10 f_{0}$.

The FFT focusing error $\varepsilon_{j}$ of the data interpolation method [5] and the data reconstruction method at different frequencies are shown in Fig.1. Obviously, the error of FFT interpolation is larger than that of data reconstruction, which is the fundamental reason why the performance of MVDR is not as good as that of data reconstruction using FFT interpolation.Figure 2 shows the FFT interpolation method and data reconstruction method in different signal to noise ratio resolution probability. It can be seen that with the improvement of signal-to-noise ratio, the resolution probability of the two methods becomes 1 , and the resolution probability of the data reconstruction method is better than that of the FFT method when the SNR is less than 10dB.Figure 3 and Figure 4, respectively, compared under different conditions of signal to noise ratio, using data reconstruction methods and FFT interpolation method and the azimuth angle of the root mean square error.It can be seen from Fig.3 and Fig.4 that the RMS error of the azimuth obtained by the data reconstruction method is less than that of the FFT interpolation method at different SNRs. Therefore, for the broadband MVDR algorithm, the data reconstruction method has better azimuth estimation performance than the FFT interpolation method. 


\section{Conclusion}

In this paper, the time domain data reconstruction method is introduced into the space array data, and the practical steps of the data reconstruction method are given. A focus error suitable for spatial resampling decoherence method is proposed. The results show that the performance of the data reconstruction method is better than that of the FFT method for the wideband MVDR algorithm, which is compared with the FFT interpolation method and the data reconstruction method by computer simulation.

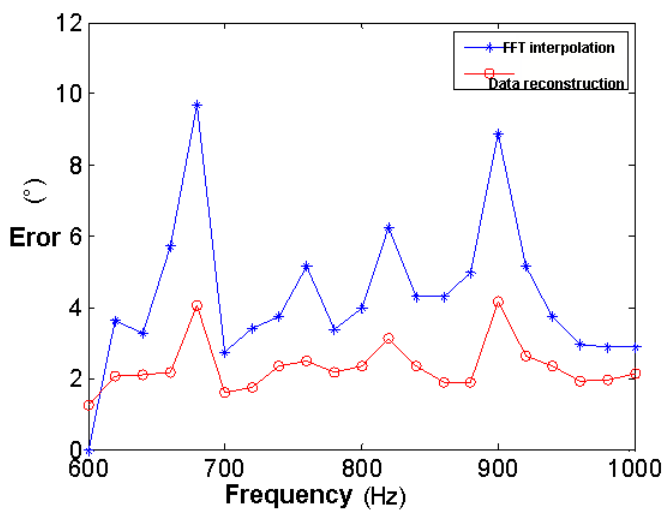

Fig.1. Frequency with the SNR curve

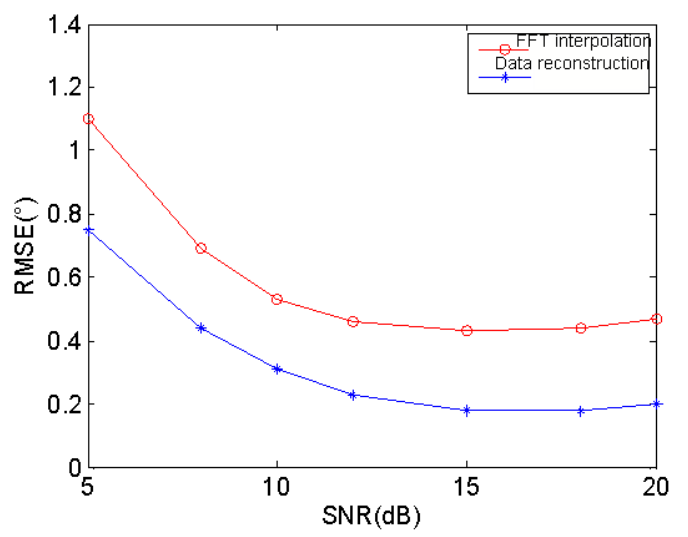

Fig.3. $-2 \circ$ Azimuth mean square error curve

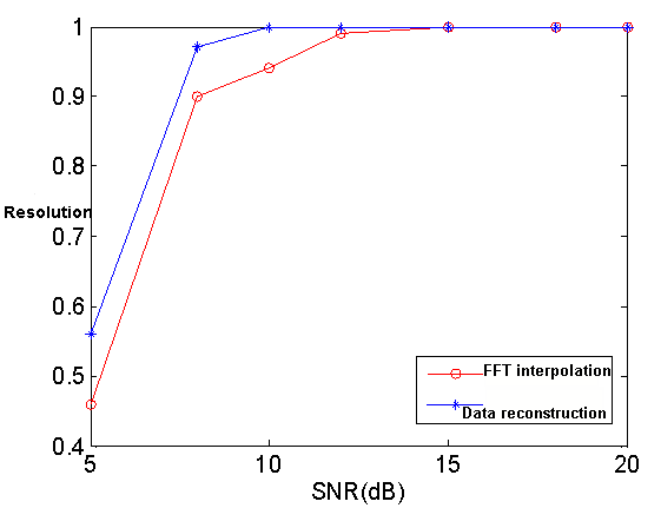

Fig.2. Resolution probability with SNR curve

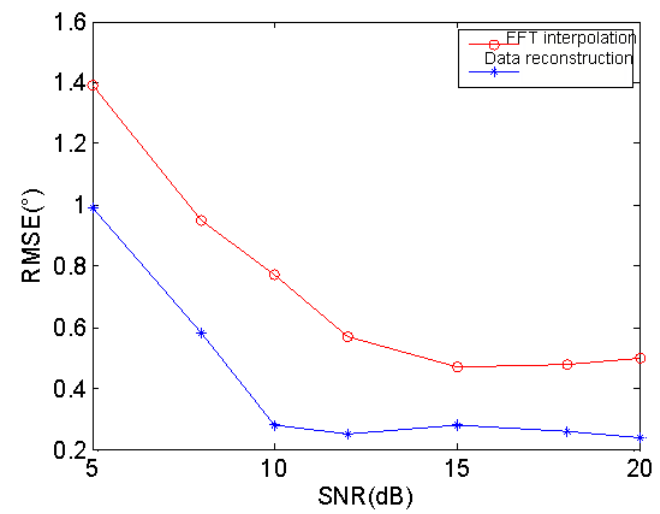

Fig.4. $4^{\circ}$ Azimuth mean square error curve

\section{References}

[1] Wax. M.s, Tie-Jun, and T.Kailath Spatio-temporal spectral analysis by eigenstructure methods[J]. IEEE. Trans. on Acoustics, Speech and Signal Processing,1984, 32(4), 817-827 .

[2] Wang. H, Kaven M. Coherent signal-Subspace Processing for the Detection and Estimation of Angles of Arrival of Multiple Wideband Sources[J]. IEEE. Trans. on Acoustics, Speech and Signal Processing, 1985, 33(4): 823-831.

[3] M.A. Doron, E. Doron, A.J. Weiss. Coherent wideband processing for arbitrary array geometry[J]. IEEE. Trans. Signal Processing, 1993, 41(1): 414-417.

[4] B. Friedlander, A.J. Weiss. Direction finding for wide-band signals using an interpolated array, 1994, 42(10): 2549-2559.

[5] Wang H, Kaveh M. Coherent signal-subspace processing for the detection and estimation of angles of arrival of multiple wide-band sources[J]. Acoustics Speech \& Signal Processing IEEE Transactions on, 1985, 33(4):823-831.

[6] Schmidt R.O. Multiple Emitter Location and Signal Parameter Estimation[J]. IEEE. Trans. 1986, 34(3): 276-280.

[7] Roy R, Kailath T. ESPRIT-a subspace rotation approach to estimation of parameters of cissoids in noise [J]. IEEE. Trans. on ASSP. 1986, 34(10):1340-1342.

[8] Pham T, Sadler B M. Adaptive wideband aeroacoustic array processing[C]// Statistical Signal and Array Processing, 1996. Proceedings. IEEE Signal Processing Workshop on. IEEE, 1996:295-298. 